\title{
Double-Rotor Switched Reluctance Machine (DRSRM): fundamentals and magnetic circuit analysis
}

\begin{abstract}
A novel switched reluctance machine with a double rotor configuration is introduced in this paper. The proposed design is based on optimization of the electromagnetic forces, which leads to a better electro mechanical energy conversion process. Finite Element Analysis (FEA) is used to simulate the torque characteristics and the harmonic distortion is used to evaluate the performance of the proposed motor. In the conventional machine structure the torque ripple is major drawback as the majority of the forces produced are in the radial direction that in turn does not contribute much to the torque production. However a larger torque value can be achieved if the normal forces are in the direction of motion. Double rotor exploits the fact that the same excitation produces dual magnetic paths if the reluctance between iron and air is reduced to half with increased area of flux linkage thereby maximizing the generated torque. Because of the difference in value in the radii the torque developed is not doubled due to the mechanical tolerances. However, the torque generated in double rotor is higher than conventional structures because of the reduced variable reluctance in the air gap. Both rotors are fixed to the same shaft which leads to a unidirectional torque and consequently by law of superposition, the torque generated piled up together. Based on the above motivation, the Double Rotor SRM (DRSRM) is proposed. Another advantage of the proposed DRSRM is the increase of the effective area usage for the machine structure. The results of our investigations indicate that the proposed geometry offers superior performance with improved torque characteristics with reduced THD.
\end{abstract}

Keyword: Double rotor; Reluctance; THD; Torque shaping 